\title{
EDITORIALS
}

\section{ASSESSMENT, ETHICS, AND THE RANDOMIZED CONTROLLED TRIAL}

\author{
Michael Baum \\ King's College School of Medicine and Dentistry, \\ Rayne Institute
}

\begin{abstract}
Decision-making in surgical practice is a highly complex interaction between the client (patient) and his or her professional advisor (surgeon). The client approaches the professional advisor full of expectations and fears. The expectations may be realistic or unrealistic, and, in the same way, the fears may be justified or unjustified. Furthermore, the client may have a variety of priorities not immediately apparent to the surgeon and may wish to express different degrees of autonomy. For example, some patients may demand the final say in determining the balance between length and quality of survival, whereas others would be happy to allow complete abrogation of their responsibilities to the surgeon, who is invited to make the difficult decisions concerning the patient's utilities. From the viewpoint of the surgeon, the transaction is equally complex, and his or her decisions may be constrained by resource allocation and time available. However, in the final analysis, assuming a beneficent doctor, the most appropriate decision will be determined by the weight of evidence that can be adduced in favor of any intervention.
\end{abstract}

There is a hierarchy that can be constructed to describe the quality of evidence in favor of any given procedure, the pinnacle of which is marked by the reproducible randomized controlled trial (5); however, to achieve this pinnacle, surgeons working in the last decades of the twentieth century have to overcome enormous ethical and financial barriers. The organization of randomized trials and other forms of technology assessment is very expensive. Yet this activity lacks sufficient glamour to call upon adequate funding from government and charitable organizations. Paradoxically, it is easier to raise money to find the cure for cancer in rats than to demonstrate that extrapolation of these laboratory findings might also be beneficial to humanity. I would, therefore, like to make the plea that the same order of funding become available to demonstrate efficacy of treatments among human subjects, as in the generation of new ideas from the basic sciences and the biological institutes.

As urgent as it may be to convince funding agencies of the importance of technology assessment, there is a greater urgency to educate the profession and lay public alike in these areas. The lay public must appreciate that, just as randomized controlled trials demonstrate the ability of a new treatment to improve length and quality of survival, the same element of methodology protects their long-term interests by being 
the most efficient way of demonstrating unpredicted hazards and toxicity. Over the last 10 or 20 years, in the United States and, in more recent years, in the United Kingdom, there has been a fashionable swing in our ethical model of medical practice, so that the patient's desire to express autonomy is now considered preeminent, trumping all other ethical premises. This expression of autonomy is best understood in the context of informed consent. Unfortunately, the expression of a patient's informed consent has been simply interpreted as the right of the patient to determine his or her own treatment when faced with several options. The public also needs to be informed about the hazards of the unscientific application of new treatments and the virtues of the randomized controlled trial. To date, the informed consent procedure has seriously inhibited the success of large randomized controlled trials and has led to the collapse of some very well-intentioned studies (4) and to the practice of relying on countries to carry out essential clinical research in which investigators are less fettered by state or federal legislation (1). At the same time, the unfettered autonomy of physicians, especially of those in the private sector, has allowed them to gratify falsely the patient's whims and expectations by implying certainties in their decision-making and of fering treatment with unfounded confidence. Again, the inability of many physicians to recognize their own uncertainties or to impart these uncertainties to their clients has made the practice of randomized controlled trials in some areas virtually impossible (8).

If we are to make serious progress in the future in a safe and scientific manner, then perhaps we should challenge either the patient's or physician's autonomy. As it is politically and perhaps morally unacceptable to challenge the patient's right to selfdetermination in health care matters, then it is the physician's autonomy that must be limited. The solution to this dilemma is essential if we are not to repeat the mistakes of the past and if we are to make significant inroads into the betterment of care for the degenerative and malignant conditions that have so far frustrated the efforts of medical scientists. If patients' informed consent is to have any meaning, then they need to be informed about the uncertainties within which physicians practice. Their consent to randomization within a trial would then have a greater foundation of truth than their consent to ad hoc treatments promoted by smooth-talking and dignified members of the medical profession. If there is genuine uncertainty as to how to proceed, then there should be an ethical imperative imposed upon all physicians to describe this uncertainty to their patients. Thus, in the long term, it should be made as difficult for physicians to practice outside a clinical trial as it is now to practice within clinical trials.

All groups involved in this debate are attempting to claim the moral high ground, suggesting that they are more ethical or more compassionate than others who take a differing standpoint. We are at risk, therefore, of polarizing the subject beyond the reach of any intellectual bridge. This will do nothing to promote the cause of science or reduce the sum of human suffering. Surely it is more fruitful to suggest that none of the parties involved in this debate comes out completely untainted. Patients who generalize from a single negative experience have unwittingly damaged the confidence of the public in the medical profession and generated unnecessary anxiety (7). The public as a whole, by demanding their "rights" while not accepting their responsibilities, have also a lot to answer for. Ninety-eight percent of patients in the United States demand the right of autonomy and the individualization of treatment, leaving only $2 \%$ to volunteer for the sake of future generations (8). The ethicist, by insisting that the ethical imperative of autonomy supersedes all other ethical imperatives, such as 
justice and beneficence, has unintentionally delayed the answers to many important clinical questions by leading to the abandonment of vital clinical trials, the perpetuation of unproven therapies, and the delay in the implementation of promising new treatments. The clinical researchers themselves, the advocates of the randomized controlled trials, may use their beneficence as a cloak to hide their dispassionate thirst for knowledge and the promotion of their own careers. The uninvolved clinicians cannot dissociate themselves from this debate because an inadequate view of these issues or a therapeutic dogmatism frequently causes them to withhold appropriate patients from important clinical trials. Finally, the multitude of local ethical committees, with their disparate membership, fierce independence, and variable standards have added to the confusion (6).

The solution to this problem lies not in international declarations or codes of conduct, which themselves are self-contradictory (2), but instead demands a pragmatic approach where we can balance the rights and responsibilities of the individual while, at the same time, promoting the cause of science and reducing the burden of human suffering in the future. I would, therefore, like to suggest the following practical solution:

1. When clinical research is nontherapeutic or for phase I and phase II drug trials, the patients or subjects be strictly volunteers, and the issue of informed consent be noncontroversial.

2. When a group of clinicians wish to address by randomized controlled trial a minor question within a community hospital, for example, comparing two types of sutures or two types of nonsteroidal anti-inflammatory drugs, then gaining informed consent be neither traumatic to the patient or the doctor, and the morality of the study can safely be left to the local or state ethical committees.

3. It is when we come to national multicenter randomized controlled trials addressing major questions funded by national bodies, such as the Medical Research Council or the National Institute of Health, that we need a radical change of emphasis. I would advocate a single national ethical committee with both professional and lay members. Appointment to this committee should be seen as a singular honor and the members should all be knowledgeable about the principles and practice of medical ethics. Once a major trial has been funded and then approved by this national ethical body, it should be launched with public notices in scientific journals and the news media. All relevant patients should then be given the right to informed consent either to enter or not to enter this national trial. In practice there would be only one nationally approved consent form for all patients being treated for the condition, either within or without the trial.

4. All patients should retain the option to abrogate their responsibility, leaving the clinician to make the final decision that might, indeed, be determined at random. Inevitably this will make increasing demands on patients at a time when they are weak and vulnerable. But, bearing in mind that we almost all become patients before we die, this is a burden that we have to accept, unless we wish to return to the dark ages when treatment was determined by intuition or conceptual rationalism rather than scientific method.

\section{REFERENCES}

1. Angell, M. Ethics in international collaborative clinical research. New England Journal of Medicine 1988, 319, 1081-83.

2. Baum, M. Do we need informed consent? Lancet, 1986, 2, 911-12. 
3. Baum, M., \& Houghton, J. Informed consent and controlled trials. Lancet, 1988, 2, 1194.

4. Faulder, C., Peckham, M. J., Baum, M., Bullimore, J. A., Cuzick, J., Durrant, K. R., Maguire, G. P., Sloane, J., Teasdale, C., Tobias, J. S., \& White, H. Informed consent: Ethical, legal and medical implications for doctors and patients who participate in randomised clinical trials. British Medical Journal, 1983, 286, 1117-21.

5. McPeek, B., Mosteller, F., \& McKneally, M. Randomized clinical trials in surgery. International Journal of Technology Assessment in Health Care, 1989, 5, 317-332.

6. Marshall, T., \& Moodie, P. Scrutinising research ethics committees. British Medical Journal, 1988, 297, 753.

7. Thomas, E. Informed consent. Lancet, 1986, 2, 1280.

8. Wittes, R. E., \& Friedman, N. A. Accrual to clinical trials. Journal of the National Cancer Institute, 1988, 80, 884-85.

\title{
EXPENSIVE MEDICAL AND SURGICAL TECHNOLOGY
}

\author{
Douglas Black \\ Royal College of Physicians of London
}

The very title - "Expensive Medical and Surgical Technology"- expresses in coded form a myth that is widely prevalent among those who consider the political and economic aspects of health care. Strangely, it is a myth that finds favor mainly at the extreme ends of the range of attitudes toward health care. Monetarists see it as an incentive toward increasing private provision of health care, while extreme egalitarians see it as another example of unjust privilege. The content of the myth is that there is a definable group of costly procedures, which can somehow be isolated from the general practice of medicine and surgery, after which such procedures can either be made the subject of special private provision or, alternatively, discarded from a rationed system of health care.

This myth is dangerous (1) and contains significant fallacies:

1. It implies that there is a category of technology that is somehow separable from the rest of medical and surgical practice.

2. It takes full account of the cost of process, while leaving out of the account the value of outcome.

3. It exaggerates the contribution made by so-called high technology to the total cost of health care.

4. It suggests that specious forms of managerial control are possible and important, thereby undermining the professional approach to the definition of priorities and the delivery of health care.

Pervasiveness and Continuity of Technology. There are two reasons why we should not isolate a particular group of procedures labeled "expensive medical and surgical techniques." First, technology is only a part of the practice of medicine. It varies in 